\title{
Doctors as institutional entrepreneurs - leading quality improvement in clinical contexts
}

\author{
Authors: Michael Page ${ }^{A}$ and Michael Smets ${ }^{B}$
}

\section{Introduction}

Implementing quality improvement (QI) in clinical contexts is notoriously challenging, with many initiatives delivering little or no improvement in practice. ${ }^{1,2}$ While the reasons for this are likely to be complex, Easton highlights the role of established organisational culture in frustrating clinicians' efforts to improve healthcare processes. ${ }^{3}$ Thus, unlike in manufacturing contexts, where process improvement typically involves operating more efficiently within 'the rules of the game, ${ }^{4}$ QI in healthcare is typically a countercultural activity, which challenges existing rules, norms and beliefs. We were interested, therefore, in how doctors function in order to deliver QI in clinical contexts.

\section{Materials and methods}

Given our interest in leadership of QI as a social phenomenon, an interpretivistic methodological approach drawing on the principles of grounded theory ${ }^{5}$ was chosen. Key informant interviews ${ }^{6}$ were conducted with a purposive sample of doctors, including former RCP chief registrars, Darzi fellows and doctors with strategic QI roles locally and nationally.

\section{Results}

It was apparent from interview data that there are cultural norms operating in healthcare settings that make successful leadership of QI initiatives challenging. There was a strong sense of individual professional autonomy amongst doctors, and it was also clear that powerful individuals could exert significant influence over their colleagues.

Doctors who had led QI used a number of strategies to tackle established cultural norms, with networking activity often proving the most successful approach. At times this involved them developing their own networks as a means of building social and political capital, while at other times it meant acting as a network broker on behalf of others and/or between powerful individuals and groups.

\section{Discussion}

Doctors who sought to implement QI in healthcare settings typically found that they were engaged in a largely counter-cultural activity. They therefore operated as institutional entrepreneurs-actors who display institutional agency by instigating change. Institutional

Authors: ${ }^{\mathrm{A}}$ Queen Mary, University of London; ${ }^{\mathrm{B}}$ The University of Oxford agency denotes "episodic forms of power" in which agents "mobilise resources, engage in institutional contest over meanings and practices, develop, support or attack forms of discourse or practiceall involving discrete, strategic acts of mobilisation, 8 Networking was a commonly-used strategic act. Yet, QI methodologies, not least Langley et al's Model for Improvement, ${ }^{9}$ often describe a model that treats the organisational context as apolitical or culturally inert. Theorising QI as a countercultural activity gives greater insight into the challenges with which QI leaders are faced than do traditional, technical-rational models of QI, and is more likely to indicate useful approaches to leading QI in these settings.

\section{Conclusion}

Currently, doctors who successfully implement QI often do so within a cultural context that is not ready to embrace QI, and powerful individuals and groups can act to derail their efforts. Consequently, these doctors exhibit considerable agency and function as institutional entrepreneurs in seeking to change the rules of the game.

\section{References}

1 Dixon-Woods M, Martin GP. Does quality improvement improve quality? Future Hosp J 2016:3:191-4.

2 Davidoff F, Dixon-Woods M, Leviton L, Mitchie S. Demystifying theory and its use in improvement. BMJ Qual Saf 2015;24:228-38.

3 Easton J. Why quality improvement at scale is hard in healthcare and what deep action we might take to address the issue. An opinion piece. Published online: www.ucl.ac.uk/iehc/research/ primary-care-and-population-health/research/isl/further-reading/ opinion-piece [Accessed February 2018].

4 North D. Institutions, Institutional Change and Economic Performance. Cambridge: Cambridge University Press, 1990.

5 Glaser B, Strauss A. The Discovery of Grounded Theory. Chicago: Aldine, 1967.

6 Strauss A and Corbin J. Basics of qualitative research - Techniques and procedures for developing grounded theory. 2nd edn. California: SAGE, 1998.

7 Smith ACT, Graetz FM. Philosophies of organisational change. Cheltenham, Glos: Edward Elgar Publishing Ltd, 2011.

8 Lawrence TB. Power, institutions and organizations. In Greenwood R, Oliver C, Sahlin K and Suddaby R (eds). Sage handbook of organizational institutionalism. London: Sage, 2008:170-97.

9 Langley G], Moen R, Nolan KM et al. Changes that result in improvement. In: The improvement guide: a practical approach to enhancing organizational performance. 2nd edn. San Francisco: Jossey-Bass, 2009:15-25. 